\title{
A double blind randomised controlled trial comparing standard dose of iron supplementation for pregnant women with two screen-and-treat approaches using hepcidin as a biomarker for ready and safe to receive iron
}

Amat Bah ${ }^{1 *}$, Rita Wegmuller ${ }^{1}$, Carla Cerami ${ }^{1}$, Lindsay Kendall ${ }^{2}$, Sant-Rayn Pasricha ${ }^{3}$, Sophie E. Moore ${ }^{4}$ and Andrew M. Prentice ${ }^{1}$

\begin{abstract}
Background: Until recently, WHO recommended daily iron supplementation for all pregnant women $(60 \mathrm{mg} / \mathrm{d}$ iron combined with $400 \mathrm{ug} / \mathrm{d}$ folic acid) where anaemia rates exceeded $40 \%$. Recent studies indicate that this may pose a risk to pregnant women. Therefore, there is a need to explore screen-and-treat options to minimise iron exposure during pregnancy using an overall lower dosage of iron that would achieve equivalent results as being currently recommended by the WHO. However, there is a lack of agreement on how to best assess iron deficiency when infections are prevalent. Here, we test the use of hepcidin a peptide hormone and key regulator of iron metabolism, as a potential index for 'safe and ready to receive' iron.

Design/Methods: This is a 3-arm randomised-controlled proof-of-concept trial. We will test the hypothesis that a screen-and-treat approach to iron supplementation using a pre-determined hepcidin cut-off value of $<2.5 \mathrm{ng} / \mathrm{ml}$ will achieve similar efficacy in preventing iron deficiency and anaemia at a lower iron dose and hence will improve safety. A sample of 462 pregnant women in rural Gambia will be randomly assigned to receive: a) UNU/UNICEF/WHO international multiple micronutrient preparation (UNIMMAP) containing $60 \mathrm{mg} / \mathrm{d}$ iron (reference arm); b) UNIMMAP containing $60 \mathrm{mg} / \mathrm{d}$ iron but based on a weekly hepcidin screening indicating if iron can be given for the next 7 days or not; c) or UNIMMAP containing $30 \mathrm{mg} / \mathrm{d}$ iron as in (b) for 12 weeks in rural Gambia. The study will test if the screen-and-treat approach is non-inferior to the reference arm using the primary endpoint of haemoglobin levels at a non-inferiority margin of $0.5 \mathrm{~g} / \mathrm{dl}$. Secondary outcomes of adverse effects, compliance and the impact of iron supplementation on susceptibility to infections will also be assessed.

(Continued on next page)
\end{abstract}

\footnotetext{
* Correspondence: Amat.Bah@lshtm.ac.uk

${ }^{1}$ MRC Unit The Gambia \& MRC International Nutrition Group, PO Box 273,

Banjul, The Gambia

Full list of author information is available at the end of the article
}

\section{$\int$ Biomed Central}

(c) 2016 The Author(s). Open Access This article is distributed under the terms of the Creative Commons Attribution 4.0 International License (http://creativecommons.org/licenses/by/4.0/), which permits unrestricted use, distribution, and reproduction in any medium, provided you give appropriate credit to the original author(s) and the source, provide a link to the Creative Commons license, and indicate if changes were made. The Creative Commons Public Domain Dedication waiver (http://creativecommons.org/publicdomain/zero/1.0/) applies to the data made available in this article, unless otherwise stated. 
(Continued from previous page)

Discussion: This trial is expected to contribute towards minimising the exposure of pregnant women to iron that may not be needed and therefore potentially harmful. If the evidence in this study shows that the overall lower dosage of iron is non-inferior to $60 \mathrm{mg} /$ day iron, this may help decrease side-effects, improve compliance and increase safety. The potential for the use of hepcidin for a simple point-of-care (PoC) diagnostic for when it is most safe and effective to give iron may improve maternal health outcomes.

Trial registration: ISRCTN21955180

Keywords: Pregnancy, Hepcidin, Anaemia, UNIMMAP, Lower dose iron, Iron deficiency

\section{Background}

Anaemia is a global public health problem affecting all population groups, but especially pregnant women and young children [1]. For pregnant women, the consequences of anaemia include mortality, poor pregnancy and birth outcomes including premature delivery, low birth weight and increased perinatal mortality [1-3]. The most significant contributor to the onset of anaemia is iron deficiency [1]. The World Health Organisation (WHO) estimates that iron deficiency anaemia (IDA) affects almost half of the world's pregnant women and pre-school children with a prevalence of over $65 \%$ in Africa and Asia, and that it causes (directly or indirectly) one fourth of all maternal deaths [3]. In The Gambia, iron deficiency anaemia among women and children has been found to be high and of public health significance with $73 \%$ of pregnant women and $56 \%$ of lactating women being anaemic [4].

Although iron deficiency with or without anaemia has important consequences for human health and child development, there has been an absence of international agreement on how best to assess the iron status of populations. Serum ferritin (SF) is one of the few biochemical indices of which low levels reflect depleted iron stores $[5,6]$ but it is known to be raised by infection and inflammation as it is an acute phase protein and thus has very high false negative rates in least developed countries [7]. Similar problems also arise with the other commonly used iron status indicators as summarised in Table 1 below.

Hepcidin is a peptide hormone that has been shown recently to be the master regulator of iron absorption and distribution in humans [8-11]. The potential for hepcidin as a superior marker for iron deficiency has been highlighted in many recent studies [12-16]. Hepcidin controls iron homeostasis by inhibiting dietary iron absorption, release of iron in the macrophages and reducing iron flow to the erythron $[8,11,17-19]$. This it does by binding to the iron exporter ferroportin inducing its internalisation and degradation [18].

Recent studies have noted that over the course of a malaria season, hepcidin integrates signals arising from parasitaemia, inflammation and anaemia $[7,20]$. The fact that hepcidin plays a crucial role in the above signals and acts both as a reporter of iron status and a regulator of iron absorption, distribution and metabolism suggests it may be the ideal index for iron deficiency and could form the basis of a PoC diagnostic for iron deficiency in at-risk population groups in developing countries [20].

WHO, originally recommended universal iron supplementation for all pregnant women with a dose of $60 \mathrm{mg}$ iron and 400ug folic acid daily [21]. However, a recent study has shown that pregnant women who are anaemic and iron deficient may be protected from malaria [22]. A recent review also indicate that pregnant women who received daily iron and folic acid supplementation are at

Table 1 Limitation of current methods of assessing IDA

\begin{tabular}{lll}
\hline Test & Measure & Limitation \\
\hline Stain bone marrow preparation & Iron stores & Expensive, invasive and traumatic \\
Haemoglobin (Hb) & Anaemia & Does not measure ID per-se \\
Serum ferritin (SF) & Iron stores & Raised by infection and inflammation \\
Zinc protopopherin (ZnPP) & Iron in new cells & Affected by infection and inflammation \\
Soluble transferrin receptor (sTfR) & Severe ID even & Affected by $(>)$ red cell prod. Lack standardised \\
& with inflammation & reference for measure' \\
STfR/logSF ratio & Iron stores & Lack standardised assay range. Ferritin affected \\
& & by infection or inflammation \\
Transferrin saturation (TSAT) & Iron levels & Affected by $(>)$ plasma concentration \\
Serum iron & Iron in sera & Affected by recent iron ingestion and infection \\
Total iron binding capacity (TIBC) & Iron in serum & Affected by infection \\
\hline
\end{tabular}


a greater risk of haemoconcentration (haemoglobin greater than $130 \mathrm{~g} / \mathrm{L}$ ) in the second and third trimester of pregnancy [23]. Although the effect of the haemoconcentration in the above review was uncertain, Ziaei et al. [24] in a randomised controlled trial (RCT) of over 700 pregnant women who took $50 \mathrm{mg}$ iron as ferrous sulphate daily found that small-for-gestational-age birth rate and the number of women with hypertensive disorders increased significantly. They concluded that routine iron supplementation in non-anaemic women is not rational and may be harmful. Recently, two hazardous complications of pregnancy; gestational diabetes mellitus and preeclampsia have been recognized to be associated with elevated body iron levels [25].

There has been little specific evidence on the relationship between risk of malaria and other infections with iron status and iron supplementation in pregnant women. Yet, the benefits of iron supplementation must be carefully weighed against the risks in developing countries [26, 27].

There is now some evidence that smaller doses of $30 \mathrm{mg}$ iron daily could achieve similar results as the daily $60 \mathrm{mg}$ iron [21]. A Cochrane review on the treatments of iron deficiency anaemia in pregnancy [28] indicated that daily low dose iron supplementation may be effective at treating anaemia in pregnancy with fewer gastrointestinal side effects compared with higher doses. WHO has now recommended the use of doses between 30 and $60 \mathrm{mg}$ for daily supplementation for pregnant women [29]. Further evidence suggest that the use of multiple micronutrient supplements with three or more micronutrients is associated with a $39 \%$ reduction in maternal anaemia compared with placebo or with two micronutrients or fewer (relative risk $0.61,95 \%$ CI 0 . $52-0 \cdot 71)$. Multiple micronutrient supplementation is also known to result in a decrease in the risk of lowbirth weight babies $(0.83,0.76-0.91)$ and small-forgestational-age babies $(0 \cdot 92,0 \cdot 86-0 \cdot 99)$ [30].

In this proof of concept study we aim to test the hypothesis that a screen-and-treat approach to iron supplementation will achieve similar efficacy in combating ID and IDA at a lower overall dosage of iron. The assumption that lower doses will improve safety and tolerability will also be tested. The design will establish whether using screen-and-treat with UNIMMAP containing either $60 \mathrm{mg}$ or $30 \mathrm{mg}$ iron per day is noninferior to UNIMMAP containing $60 \mathrm{mg} /$ day as a universal daily supplement.

\section{Design/Methods}

\section{Study design}

This study is designed as a proof-of-concept, 3-arm, double blind, RCT over a period of 12 weeks with a sample of 462 pregnant women randomly assigned to receive: a) UNIMMAP containing $60 \mathrm{mg} /$ day iron; b)
UNIMMAP containing $60 \mathrm{mg} /$ day iron but based on a weekly hepcidin screening indicating if iron can be given for the next 7 days or not; c) UNIMMAP containing $30 \mathrm{mg} /$ day iron based on screening as in (b).

\section{Determining the hecidin cut-off value}

The hepcidin cut-off of $<2.5 \mathrm{ng} / \mathrm{mL}$ as a threshold (to receive iron or not) is based on the analysis of sera from 270 pregnant women participating in the ENID study [31] with samples available for 3 time points (12-14 weeks, 20 weeks and 30 weeks gestation). A receiver operating characteristics (ROC)-curve was generated to measure the area under the curve $\left(\mathrm{AUC}^{\mathrm{ROC}}\right)$. Method described elsewhere [12].

\section{Study location and participants}

The Hepcidin and Anaemia in Pregnancy (HAPn) study will be carried out in 12 communities of Jarra West and Kiang East (rural Gambia) about $150 \mathrm{~km}$ from the capital city of Banjul. The Regional Health Team, the health facilities within the study area and the individual communities have been sensitised and their approval gained for conducting the study.

The study will involve 462 healthy pregnant women between the ages of 18 and 45 years (established by asking, use of birth certificates, identity cards or calendar events) who are pregnant (estimated at between 14 and 22 weeks gestation, by fundal height assessment and date of last menstrual period (LMP)) and are likely to be resident in the study area for the duration of the study period.

Pregnant women who are identified as potential participants will be excluded from the study if found to be (i) severely anaemic $(<7 \mathrm{~g} / \mathrm{dL})$, (ii) seriously ill (infectious disease of clinical significance) at recruitment (iii) suffer from a chronic disease (iv) have pregnancy complications (e.g., pre-eclampsia) at enrolment or (v) already participating in another study.

\section{Detailed study procedure}

Screening and enrollment (Baseline)

Pregnant women living within the two health facility catchment areas will be identified as they visit the Reproductive and Child Health $(\mathrm{RCH})$ clinics to register and book their pregnancies. As part of the routine services provided, a nurse midwife determines their stage of pregnancy. If a woman is within the window of the study (14-22 weeks gestation), she will be invited by the study team to take part in the study and informed consent will be sought. Once a signed informed consent is obtained and all of the inclusion and none of the exclusion criteria are met, she will be enrolled in the study, and asked to provide $5 \mathrm{~mL}$ venous blood (Day 0 below). Participants will thereafter be assigned to one of 
the 3 study groups (see randomisation, below). All women enrolled in to the study will be provided with long lasting insecticide-treated bed nets (LLINs).

\section{Follow-up}

Following recruitment, women will be followed up weekly in their communities. Each week, trained MRC field assistants (FA) will invite the study participants to a central location within their communities for collection of a finger prick blood sample for analysis of haemoglobin $(\mathrm{Hb})$ using a HemoCue $\mathrm{Hb} 301$ analyser (HemoCueAB, Angelholm, Sweden), malaria parasitaemia using a SD Bioline One step malaria antigen Pf Test (SD Standard Diagnostics, Inc. Kyonggi-do, Korea) and hepcidin levels using the BACHEM Hepcidin-25 ELISA. Hb and malaria assessments will be performed immediately; samples for hepcidin measurements will be transferred on ice to a laboratory at MRC Keneba where analysis will commence within the hour of arrival. The following day hepcidin results will be available and a 7 day supply of supplements packed according to the hepcidin results (computer generated). The day after, participants will be provided with their supplements. While the supplements are being distributed, the FA will also assess beneficial effects, adverse events and compliance. All activities will be documented on a case report form (CRF) using electronic data capture in the form of a hand held device (SAMSUNG Galaxy Tab3 Model SM-T211). Data will be sent through a secure internet connection to the MRC database.

\section{Ethics and safety monitoring}

The trial has been approved by the Medical Research Council (MRC) Scientific Coordinating Committee (SCC) and the Joint Gambia Government MRC Ethics Committee. It will be overseen by a Data Safety Monitoring Board (DSMB) and a Trial Steering Committee assisted by a Trial Monitor (TM). Together they will be responsible for reviewing all interim data, treatment safety and efficacy including the protection of the rights and wellbeing of the participants. The trial will be conducted according to Good Clinical Practice (GCP) principles taking in to consideration the provisions of the World Medical Association (WMA) Declaration of Helsinki (October 2013).

Participants will be monitored on each scheduled follow up day for all adverse events (AEs) defined as any untoward or unfavourable medical occurrence in a human subject, including signs and symptoms which are temporally associated with the research procedure or trial intervention, whether or not considered related to the subject's participation in the research. All serious adverse events (SAEs) defined as any AE that is life-threatening or results in death or require hospitalisation or prolongation of hospitalisation, is a persistent or significant disability/ incapacity or is a congenital anomaly/birth defect or a reported maternal death, miscarriage or stillbirth will be recorded as SAEs and investigated by a physician. Monitoring of the participants will then continue until they deliver and the outcome of the pregnancy for both mother and child is known (postnatal check-up within $72 \mathrm{~h}$ after delivery).

\section{Collection and analyses of biological samples during enrollment and follow-up visits}

As described, finger prick blood samples will be collected weekly. Additional $5 \mathrm{~mL}$ venous blood samples will also be collected at 4 different time-points (Days 0, 14, 49 and 84) within the 12 week period of the study. As intermittent preventative treatment (IPT) is routine in this region, participants will receive their IPT dose immediately after blood draws are done at days 14 and 49 in order not to influence our ex vivo P. falciparum assays. All venous blood draws will be carried out by the study nurse and finger prick blood samples by the field assistants (FAs).

Full haematology including haemoglobin and reticulocytes will be assessed on samples collected on Days 0, 14, 49 and 84 in a $1.2 \mathrm{~mL}$ EDTA Sarstedt tube using the Medonic M Series analyser.

Biochemistry analysis of plasma ferritin, iron, transferrin saturation (TSAT), soluble transferrin receptor (sTfR), C-reactive protein (CRP), and alpha-1-acid glycoprotein (AGP) will be measured by Cobas Integra 400+ using $500 \mu \mathrm{l}$ from -20 frozen samples. The Cobas measuring principle for ferritin, transferrin and CRP will be via turbidimetric principle at $552 \mathrm{~nm}, 340 \mathrm{~nm}$ and $552 \mathrm{~nm}$ respectively and for iron, FerroZine method without deproteinization. sTfR will be measured photometrically at $583 \mathrm{~nm}$ and alpha-1-acid glycoprotein will be through turbidimetric. $\mathrm{Hb}$ genotyping will be performed using $\mathrm{Hb}$ electrophoresis with Shandon Vokam 400 on all samples collected on Day 0.

This study is not powered to use clinical endpoints to assess safety. Instead the trial will use in vitro assays to assess safety on each of the four venous samples per subject. Ex vivo growth of P. falciparum will be assessed in washed red blood cells (RBCs) using a field-ready 96well plate method with florescence-activated cell sorting (FACS) readout [32]. A small subset of RBCs will be lysed for measurement of riboflavin status by the erythrocyte glutathione reductase activation coefficient (EGRAC) test because this may affect RBC stability.

The ex vivo growth of sentinel organisms (Escherichia coli, Yersinia enterocolitica, S. enterica serovar (Typhimurium), Staphylococcus epidermidis, Staphylococcus aureus and Candida albicans) analyses will be performed in frozen $\left(-20{ }^{\circ} \mathrm{C}\right)$ plasma $(400 \mu \mathrm{l})$ as previously described in the investigation of the effects of 
iron supplementation on pathogen virulence in human serum [33].

DNA will be extracted from baseline whole blood samples to study the genes implicated in iron metabolism. Known genetic risk factors for malaria assays will include alpha-thalassemia, G6PD and sickle traits. Furthermore, putative functional and key tagging variants in iron regulatory and inflammatory pathways will be screened.

\section{Randomisation and blinding}

\section{Randomisation}

Recruited women will be randomly assigned (computer generated) to one of the 3 treatment arms (equal number in each arm) balanced by the $\mathrm{Hb}$ concentration of the baseline blood sample and gestational age. To achieve this, at each day of recruitment, subjects will be categorised into two $\mathrm{Hb}$ classes (above and below the median $\mathrm{Hb}$ of the respective day) and according to 2 gestational age periods (14-18 weeks, 19-22 weeks) making 4 classes. In each of the 4 classes, the women will be randomly assigned to the 3 treatment arms using a predetermined block randomisation.

The randomisation database of treatment arms (A, B, C) will be password protected with the database developer and his assistant knowing the password. If a subject needs to be unblinded at the request of the DSMB, their treatment can be easily identified without unblinding the whole study.

\section{Blinding}

Participants, field workers, study nurse and PI will be blinded as to which treatment group participants belong to and which supplement participants receive each week. The supplements will be pre-packed on a weekly basis by the field coordinator in Keneba using lists automatically (computer) generated by the data office taking into account the hepcidin results of the participants. The list will indicate the letter of the supplement the participant receives for the following 7 days but the field coordinator will not know which code is allocated to which supplement. The capsules are coded ( 2 codes for treatment arm A (60 mg and $60 \mathrm{mg}$ iron), 2 codes for arm B (60 $\mathrm{mg}$ and $0 \mathrm{mg}$ iron) and 2 codes for arm C (30 mg and $0 \mathrm{mg}$ iron), see Fig. 1 . The pre-packed weekly supplies labelled with each participant's ID will be handed over to the PI who will be responsible for handing them over to the field workers who will distribute to the individual participants. The laboratory staff and data entry clerks will also be blinded.

The allocation of the colour code will be done by 2 people independent of the study and the key will be kept in a locked cabinet in Keneba. The blinding for the study may be broken if in any of the 3 treatment arms, safety issues arise and the trial team is advised by the DSMB to do so.

\section{Investigational product}

The investigational product to be used is the UNICEF/ WHO/UNU international multiple micronutrient preparation (UNIMMAP). Three products will be administered (UNIMMAP with $60 \mathrm{mg}$ iron, UNIMMAP with $30 \mathrm{mg}$ iron, UNIMMAP with $0 \mathrm{mg}$ iron). All formulations also contain 400 ug folic acid and 14 other micronutrients (Table 2). The UNIMMAP supplement has already been used safely in other pregnancy trials [34]. The formulations are produced by DSM South Africa under Good Manufacturing Practice (GMP) conditions where it will also be dosed into gelatin capsules and packed in tubs. The labelling will include a statement that 'trial medications are only for use of trial participant'.

The products will be stored under controlled conditions (in an air-conditioned storage at around $20^{\circ} \mathrm{C}$ ) at MRC Keneba. The product is stable for 18 months if kept under these conditions.

Each participant will receive 1 capsule per day. Each week field workers will be visiting study participants to distribute the respective weekly supply (7 capsules) to each study participant. The participants will be instructed to take 1 capsule a day with water or another drink. Each time the field workers distribute the new weekly supply of capsules they will account for the number of capsules consumed/not consumed from the previous week in order to check for compliance. Any left-over capsules will be collected by the field workers.

\section{Sample size and statistical analysis plan Sample size determination}

Using the haemoglobin data obtained from pregnant women enrolled in the ENID study in West Kiang [31], a SD of 1.28 was derived. This was used to obtain a sample size of 154 participants for each of the 3 arms

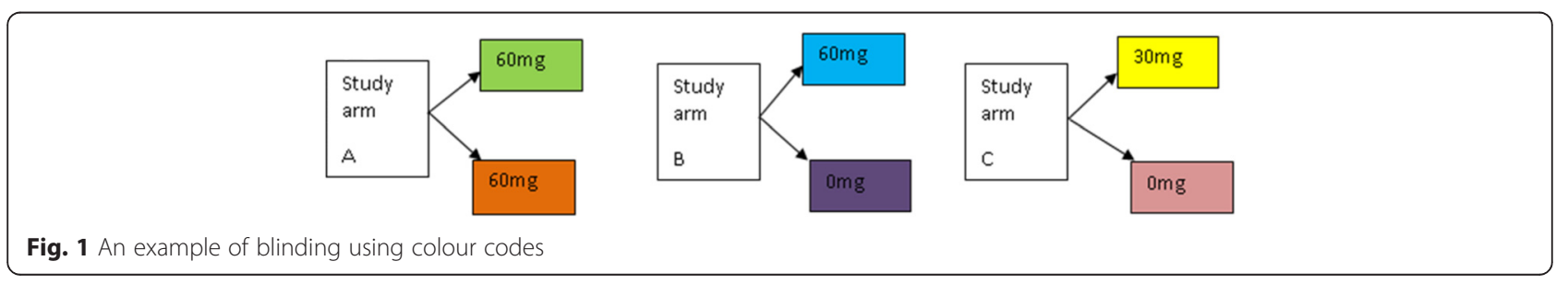


Table 2 Intervention product - Formulation based on UNU/ UNICEF/WHO supplement called UNIMMAP

\begin{tabular}{ll}
\hline Micronutrients & Dose/day \\
\hline Vitamin A (ug RE) & 800 \\
Vitamin D (IU) & 200 \\
Vitamin E (mg) & 10 \\
Thiamine (mg) & 1.4 \\
Riboflavin (mg) & 1.4 \\
Niacin (mg) & 18 \\
Folic acid (ug) & 400 \\
Vitamin B6 (mg) & 1.9 \\
Vitamin B12 (ug) & 2.6 \\
Vitamin C (mg) & 70 \\
Zinc (mg) & 15 \\
Iron (mg) & 60 or 30 or 0 \\
lodine (ug) & (placebo) \\
Selenium (ug) & 150 \\
Copper (mg) & 65 \\
\hline
\end{tabular}

(Table 3) calculated using a 1-sided $\alpha$ (alpha) of 2.5 with a conservative-Bonferroni type correction $(\div 3)$ so as not to inflate the type 1 error rates while performing multiple tests. A total sample size of 462 pregnant women followed up for 12 weeks with a less than $10 \%$ loss to follow-up will provide $80 \%$ power to establish that:

1) arm B is non-inferior to arm A on the primary endpoint defined below.

2) $\operatorname{arm} \mathrm{C}$ is non-inferior to arm $\mathrm{A}$ at the same level as described in the statistical analysis plan.

3) $\operatorname{arm} C$ is non-inferior to $\operatorname{arm} B$.

Note: it is being stated a priori that arm $\mathrm{C}$ will be compared with B for non-inferiority to explore if the results can influence policy on the further lowering of the dose of iron for those not iron deficient. The study (sample size) is powered for this analysis.

\section{Statistical analysis plan}

The approach to the analysis for this trial will be a test of non-inferiority. As recommended for the acceptance of

Table 3 Study arms

\begin{tabular}{llll}
\hline Group & Dose (mg/day Fe) & Universal (N) & $\begin{array}{l}\text { Screen with Hepcidin } \\
\text { (Yes or No) }\end{array}$ \\
\hline A & 60 & 154 & No \\
$B^{a}$ & 60 & 154 & Yes \\
$C^{\text {a }}$ & 30 & 154 & Yes \\
\hline
\end{tabular}

${ }^{\mathrm{a}}$ Groups $\mathrm{B}$ and $\mathrm{C}$ will be tested weekly and only given their next seven day supply of iron if plasma hepcidin falls below cut-off for 'safe and ready' non-inferiority analysis, a per-protocol (PP) analysis will be performed. Additional analysis including all $\mathrm{Hb}$ measurements (not only day 84) will be explored. These will be described in a more detail statistical analysis plan.

\section{Primary endpoint}

The primary non-inferiority endpoint is pregnancyadjusted haemoglobin at Day 84. To adjust for multiple testing (3 arms), non-inferiority will be tested with a $96.7 \%$ CI of the lower $0.83 \%(2.5 \% / 3)$ limit for the difference. The lower confidence limit for the difference in haemoglobin concentration between the universal and screened treatments on Day 84 will be above $-0.5 \mathrm{~g} / \mathrm{dL}(-5 \mathrm{~g} / \mathrm{L})$, the smallest value considered to be of minimum public health relevance. See illustration in Fig. 2.

\section{Secondary endpoints}

i. Proportion of $\mathrm{Hb}<11 \mathrm{~g} / \mathrm{dL}(\%)$ at Day 84.

ii. Hepcidin at Day 84 (as a continuous variable but also using a cut-off point of $>2.5 \mathrm{ng} / \mathrm{mL}$ to calculate the proportion).

iii. sTfR/log-ferritin ratio (ferritin index $<2.0$ ) at Day 84 (continuous variable but a proportion will also be calculated).

iv. Iron deficiency anaemia (IDA) defined as $\mathrm{Hb}<11 \mathrm{~g} /$ $\mathrm{dL}$ and ferritin $<15 \mathrm{ug} / \mathrm{L}$ when CRP is $<5 \mathrm{mg} / \mathrm{L}$ OR $\mathrm{Hb}<11 \mathrm{~g} / \mathrm{dL}$ and ferritin $<30 \mathrm{ug} / \mathrm{L}$ when CRP is $>$ $5 \mathrm{mg} / \mathrm{L}$ and ferritin index $<2.0$.

v. Iron dosage (number of weeks supplemented).

vi. Adverse events that may include nausea/vomiting, dizziness, constipation, black stool, stomach discomfort assessed weekly will be evaluated at Day 28 and Day 84. In addition, an aggregate score will also be assessed.

vii.Compliance.

viii $P$. falciparum growth in serum (difference between in vitro growth rates at Days 0, 14, 49 and 84).

ix. Ex vivo growth of sentinel bacteria (difference between in vitro growth rates at Days 0, 14, 49 and 84).

Analysis of the continuous variables (ii) and (iii) will be based on comparing means using a t-distribution where $95 \% \mathrm{CI}$ is calculated for the difference in arm A compared to $\mathrm{B}$ and $\mathrm{C}$ respectively and also between $\mathrm{B}$ and C. A logarithmic transformation will be applied to non-normally distributed variables and unpaired t-tests done on the transformed data. For variables (i), (iv), (v), (vi), (vii), (viii) and (ix), a frequency distribution using $X^{2}$ comparing proportions between the arms will be performed with a statistical test for significance for the difference between the arms set at $5 \%(P<0.05)$. Additionally, adverse events will be analysed using multiple 


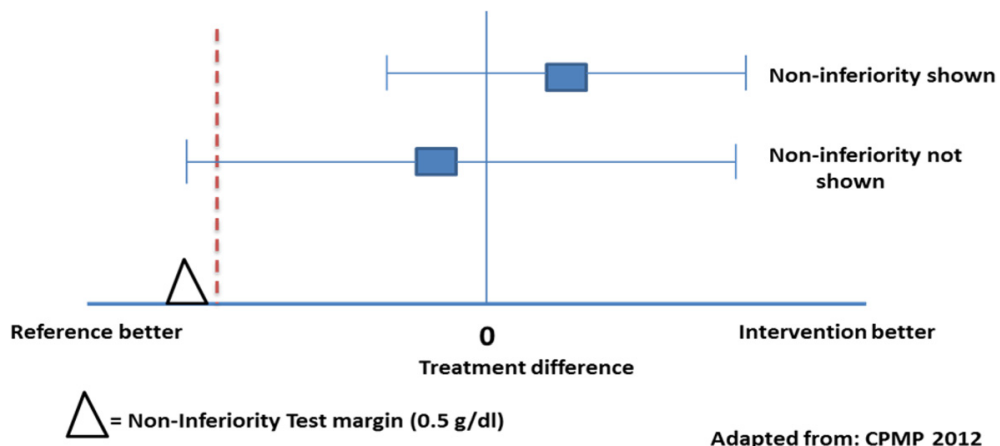

Fig. 2 Confidence interval approach to analysis of non-inferiority trial

regression analysis controlling for possible confounders to see which events or aggregate score are associated to which arm. Compliance will be assessed comparing proportions consumed in each arm with number consumed as numerator and the total number of capsules prescribed between enrolment and end of study as the denominator. STATA 12.1 or any of its latest versions will be used for all the analyses.

Further exploratory analysis will be conducted and the endpoints include:

Primary endpoints adjusted for CRP, AGP and malaria (we decided a priori that the primary endpoints will not be adjusted for the above, however, we wish as part of an exploratory analysis to adjust for them), sTfR, Ferritin, and TSAT.

\section{Informed consent and confidentiality}

All field workers taking part in the recruitment of participants will be trained on translating and issuing of the informed consent documentation. The information sheet will be translated to all the illiterate participants in a language that they understand in the presence of an independent witness. The literate participants will be allowed to read the information sheet in their own time. Participants will also be encouraged to ask questions and seek clarification from the field workers and the PI. If the participant agrees to take part in the study, informed consent is recorded by a signature or thumbprint.

Participants will be allocated an individual identification number. Participant identification numbers will be used on all samples and data forms generated during the course of the study. Following sample collection and data entry, linkage of the ID back to the study participant will not be possible without a lookup table, which will be held by the data manager only and his designated data staff during the course of the study. The field worker will have a printout version of the list. Once data collection is complete, analysis will be performed on an anonymised copy of the data. All forms and case report forms (CRF) will be kept in locked files. At all stages, staff/collaborators responsible for sample analysis will be blinded as to the subject's identification. Together, these processes will ensure complete confidentiality of the data gathered and impartiality of data analyses.

\section{Discussion}

WHO has identified the need for a lower dose in iron supplementation as recommendations being used by countries can pose risks to some pregnant women. This trial will test the efficacy of employing a screen-andtreat approach to minimise iron exposure whilst achieving a similar therapeutic effect.

As $50 \%$ of anaemia in pregnancy is assumed to be due to ID, the assessment of iron status (not only anaemia) in supplementation programmes is critical and hepcidin has shown the potential of being an improved biomarker for iron status and therefore a signal for the safe administration of iron in pregnancy. In this study we will explore this potential of hepcidin with a pre-determined cut-off value of $<2.5 \mathrm{ng} / \mathrm{ml}$ to screen for the readiness to receive iron. When iron is needed, we will supplement using oral, tablet form UNIMMAP formulation containing $60 \mathrm{mg}$ iron daily (universal) on one hand or provide 60 or $30 \mathrm{mg}$ iron daily only when hepcidin levels are below the threshold cut-off value mentioned above (screenand-treat). We hypothesise that a screen-and-treat approach to iron supplementation will achieve similar efficacy in combating ID and IDA at a lower overall dosage of iron as therapy will be targeted to periods when the enterocyte iron absorption channels are open. We assume that lower doses will improve safety and tolerability and these will be tested as secondary outcomes.

In summary, this trial will contribute towards minimising exposure to excessive iron that may not be needed and may indeed be harmful and allow the pregnant woman to maximise the absorption and utilisation of iron when it is most needed. In addition, the overall lower dosage may help decrease side-effects and increase compliance. The exploration of hepcidin as a potential for a simple PoC diagnostic to screen for the readiness 
to receive iron will assist health workers to make the right decisions for iron supplementation which will help in improving health care delivery and reduce maternal morbidity and mortality as part of efforts to meet the Millennium Development Goal 5 and the forthcoming Sustainable Health Agenda.

\begin{abstract}
Abbreviations
AGP, alpha-1 acid glycoprotein; CRF, case report form; CRP, C-reactive protein; DSM, Dutch State Mines; ELISA, enzyme-linked immunosorbent assay; FA, field assistant; FACS, florescence-activated cell sorting; FW, field worker; GMP, good manufacturing practice; $\mathrm{Hb}$, haemoglobin; ID, iron deficiency; IDA, iron deficiency anaemia; LLINs, long lasting insecticide-treated nets; MRC- ING, Medical Research Council International Nutrition Group; MRC, Medical Research Council; represents Medical Research Council Unit, The Gambia; PoC, point of care; $\mathrm{RCH}$, reproductive and child health; $\mathrm{RCT}$, andomised control trial; RDT, rapid diagnostic test; SAT, transferrin saturation; SCC, Scientific Coordinating Committee; SF, serum ferritin; sTfR, soluble transferrin receptors; TM, trial monitor; UNIMMAP, UNICEF/WHO/UNU International Multiple Micronutrient Preparation; WHO, World Health Organisation; WIMM, Weatherall Insttitute of Molecular Medicine
\end{abstract}

\section{Acknowledgement}

The trial will not be possible without the funding provided by the Bill and Melinda Gates Foundation to the MRC-ING. The trial is also supported by the UK MRC and the UK Department for International Development (DFID) under the MRC/DFID Concordant agreement. We appreciate the valuable contribution of Momodou W. Jallow and Ebrima Ceesay for analysing the blood samples for the determination of the hepcidin cut-off point used in this trial.

\section{Authors' contributions}

$A B$ is the main and corresponding author as this is part of his PhD. He put together the proposal. AMP is a substantial contributor to the concept and design of the protocol. He is also the supervisor of the PhD student. RCW is a contributor to the design of the protocol as well as reviewing the contents. SEM contributed to the reviewing of the protocol and she is also the associate supervisor of the PhD student. CC is a collaborator who is contributing to the ex-vivo analyses. LK is the statistician of the project and contributed to the statistical write-up of the protocol. SP is the collaborator who did the analysis for the hepcidin cut-off point being used and contributed to the design of the protocol. All authors read and approved the final manuscript.

\section{Authors' information}

$A B$ is the Deputy Executive Director of the National Nutrition Agency [NaNA] in The Gambia who is seconded to MRC-ING to pursue a PhD. He has a BSC in Applied Human Nutrition from Queen Margaret University, Edinburgh and an MSc in Public Health Nutrition from the LSHTM.

AMP is a Professor of nutrition at the LSHTM and Theme leader for the MRC-ING. RW is the Head of Station for Keneba Field Station MRC Unit The Gambia. SEM is Group Leader, Maternal and Child Helath Group, MRC Human Nutrition Research.

CC is an Assistant Professor at the Gillings School of Global Public Health, University of North Carolina and Senior Investigator Scientist, MRC Unit The Gambia.

LK is a statistician at the MRC Gambia Unit The Gambia.

$\mathrm{SP}$ is a Fellow at Weatherall Institute of Molecular Medicine (WIMM), University of Oxford.

\section{Competing interests}

The authors declare that they have no competing interests.

\section{Author details}

${ }^{1}$ MRC Unit The Gambia \& MRC International Nutrition Group, PO Box 273, Banjul, The Gambia. ${ }^{2}$ MRC Unit The Gambia, PO Box 273, Banjul, The Gambia. ${ }^{3}$ The Weatherall Institute of Molecular Medicine, University of Oxford, John Radcliffe Hospital, Oxford OX3 9DS, UK. ${ }^{4}$ MRC Human Nutrition Research, Elsie Widdowson Laboratory, 120 Fulbourn Road, Cambridge CB1 9NL, UK.
Received: 28 July 2015 Accepted: 14 June 2016

Published online: 13 July 2016

\section{References}

1. WHO. Worldwide prevalence of anaemia 1993-2005. 2008.

2. Galloway R, Dusch E, Elder L, Achadi E, Grajeda R, Hurtado E, Favin M, Kanani S, Marsaban J, Meda N, Moore KM, Morison L, Raina N, Rajaratnam J, Rodriquez J and Stephen C. Women's perceptions of iron deficiency and anemia prevention and control in eight developing countries. Soc Sci Med. 2002;55(4):529-44

3. WHO. The World Health Report: Reducing risks, promoting healthy life. 2002.

4. Bah A, Semega-Janneh I, Prentice AM, Bates C. Nationwide survey on the prevalence of vitamin $A$ and iron deficiency in women and children in the Gambia. Banjul: National Nutrition Agency; 2001.

5. Kohgo $Y$, Torimoto $Y$, Kato J. Transferrin receptor in tissue and serum: updated clinical significance of soluble receptor. Int J Hematol. 2002;76(3):213-8.

6. Lynch S. Case studies: iron. Am J Clin Nutr. 2011;94(2):673S-8S,

7. de Mast Q, Syafruddin D, Keijmel S, Riekerink TO, Deky O, Asih PB, Swinkels DW, van der Ven AJ. Increased serum hepcidin and alterations in blood iron parameters associated with asymptomatic $P$. falciparum and $P$. vivax malaria. Haematologica. 2010;95(7):1068-74.

8. Ganz T. Hepcidin and iron regulation, 10 years later. Blood. 2011;117(17):4425-33.

9. Anderson GJ, Frazer DM, Wilkins SJ, Becker EM, Millard KN, Murphy TL, McKie AT, Vulpe CD. Relationship between intestinal iron-transporter expression, hepatic hepcidin levels and the control of iron absorption. Biochem Soc Trans. 2002;30:724-6.

10. Nicolas G, Chauvet C, Viatte L, Danan JL, Bigard X, Devaux I, Beaumont C, Kahn $\mathrm{A}$ and Vaulont $\mathrm{S}$. The gene encoding the iron regulatory peptide hepcidin is regulated by anemia, hypoxia, and inflammation. J Clin Invest. 2002;110:1037-44.

11. Huang H, Constante M, Layoun A, Santos MM. Contribution of STAT3 and SMAD4 pathways to the regulation of hepcidin by opposing stimuli. Blood. 2009;113:3593-9.

12. Pasricha SR, McQuilten Z, Westerman M, Keller A, Nemeth E, Ganz T, Wood E. Serum hepcidin as a diagnostic test of iron deficiency in premenopausal female blood donors. Haematologica. 2011;96(8):1099-105.

13. Kemna EH, Tjalsma H, Willems HL, Swinkels DW. Hepcidin: from discovery to differential diagnosis. Haematologica. 2008;93(1):90-7.

14. Heming $N$, Montravers $P$, Lasocki S. Iron deficiency in critically ill patients: highlighting the role of hepcidin. Crit Care. 2011;15(2):210.

15. Kroot JJ, Tjalsma H, Fleming RE, Swinkels DW. Hepcidin in human iron disorders: diagnostic implications. Clin Chem. 2011;57(12):1650-69.

16. Thomas C, Kobold U, Thomas L. Serum hepcidin-25 in comparison to biochemical markers and hematological indices for the differentiation of iron-restricted erythropoiesis. Clin Chem Lab Med. 2011:49(2):207-13.

17. Andrews NC, Schmidt PJ. Iron homeostasis. Annu Rev Physiol. 2007;69:69-85.

18. Ganz T, Nemeth E. Regulation of iron acquisition and iron distribution in mammals. Biochim Biophys Acta. 2006;1763(7):690-9.

19. Young B, Zaritsky J. Hepcidin for clinicians. Clin J Am Soc Nephrol. 2009:4(8):1384-7.

20. Prentice AM, Doherty CP, Abrams SA, Cox SE, Atkinson SH, Verhoef H, Armitage $\mathrm{AE}$ and Drakesmith $\mathrm{H}$. Hepcidin is the major predictor of erythrocyte iron incorporation in anemic African children. Blood. 2012; 119(8):1922-8.

21. WHO. Iron Deficiency Anaemia Assessment, Prevention, and Control. Guide for Programme Managers. 2001.

22. Senga EL, Harper G, Koshy G, Kazembe PN, Brabin BJ. Reduced risk for placental malaria in iron deficient women. Malar J. 2011:10:47.

23. Pena-Rosas JP, Viteri FE. Effects and safety of preventive oral iron or iron+folic acid supplementation for women during pregnancy. Cochrane Database Syst Rev. 2009:4:CD004736.

24. Ziaei S, Norrozi M, Faghihzadeh S, Jafarbegloo E. A randomised placebocontrolled trial to determine the effect of iron supplementation on pregnancy outcome in pregnant women with haemoglobin $\geq 13.2 \mathrm{~g} / \mathrm{dl}$. BJOG. 2007;114(6):684-8.

25. Weinberg ED. Are iron supplements appropriate for iron replete pregnant women? Med Hypotheses. 2009;73(5):714-5.

26. Sangare L, van Eijk AM, Ter Kuile FO, Walson J, Stergachis A. The association between malaria and iron status or supplementation in pregnancy: a systematic review and meta-analysis. PLoS One. 2014;9(2):e87743. 
27. Pena-Rosas JP, De-Regil LM, Dowswell T, Viteri FE. Daily oral iron supplementation during pregnancy. Cochrane Database Syst Rev. 2012;12:Cd004736.

28. Reveiz L, Gyte GM, Cuervo LG, Casasbuenas A. Treatments for iron-deficiency anaemia in pregnancy. Cochrane Database Syst Rev. 2011;10:CD003094.

29. WHO. Guideline: daily iron and folic acid supplementation in pregnant women. Geneva: World Health Organisation; 2012.

30. Bhutta ZA, Ahmed T, Black RE, Cousens S, Dewey K, Giugliani E, Haider BA, Kirkwood B, Morris SS, Sachdev HP and Shekar M. What works? Interventions for maternal and child undernutrition and survival. Lancet. 2008:371(9610):417-40.

31. Moore SE, Fulford AJ, Darboe MK, Jobarteh ML, Jarjou LM, Prentice AM. A randomized trial to investigate the effects of pre-natal and infant nutritional supplementation on infant immune development in rural Gambia: the ENID trial: Early Nutrition and Immune Development. BMC Pregnancy Childbirth. 2012;12(1):107.

32. Clark MA, Goheen MM, Spidale NA, Kasthuri RS, Fulford A, Cerami C. RBC barcoding allows for the study of erythrocyte population dynamics and P. falciparum merozoite invasion. PLoS One. 2014;9(7):e101041.

33. Cross JH, Bradbury RS, Fulford AJ, Jallow AT, Wegmüller R, Prentice A M, Cerami C. Oral iron acutely elevates bacterial growth in human serum. Scientific Reports, 5, 16670. 2015. http://doi.org/10.1038/srep16670.

34. Fall CH, Fisher DJ, Osmond C, Margetts BM. Multiple micronutrient supplementation during pregnancy in low-income countries: a meta-analysis of effects on birth size and length of gestation. Food Nutr Bull. 2009;30(4 Suppl):S533-46.

\section{Submit your next manuscript to BioMed Central and we will help you at every step:}

- We accept pre-submission inquiries

- Our selector tool helps you to find the most relevant journal

- We provide round the clock customer support

- Convenient online submission

- Thorough peer review

- Inclusion in PubMed and all major indexing services

- Maximum visibility for your research

Submit your manuscript at www.biomedcentral.com/submit

C Biomed Central 